\section{Saúde da População Negra: como nascem, vivem e morrem os indivíduos pretos e pardos em Florianópolis (SC)}

\author{
Black Population Health: how black and brown people born, live \\ and die in Florianopolis (SC)
}

\section{Salud de la Población Negra: cómo nacen, viven y mueren los individuos negros y pardos en Florianópolis (SC)}

\section{Resumo}

Objetivo: Realizar um diagnóstico situacional das condições de saúde da população negra no município de Florianópolis (SC). Métodos: Trata-se de estudo do tipo levantamento, descritivo. Os dados dos sistemas de informação em saúde foram consultados nas bases disponíveis (prontuários eletrônicos municipais, dados censitários e TABNET DATASUS estadual e nacional), com análise da variável raça/cor, no período de 2010 a 2016. Resultados: Os resultados revelam, na população negra, piores condições de escolaridade e renda, maior proporção de mães adolescentes, menor número de consultas pré-natal, maiores proporções de casos de sífilis em gestantes e tuberculose $e$ menor cobertura de plano privado de saúde. Os pacientes negros frequentaram proporcionalmente mais os Centros de Atenção Psicossocial, em comparação aos atendimentos nos Centros de Saúde. Dentre as três primeiras causas de óbitos que encurtam a vida, AIDS e homicídios aparecem todos os anos dentre a população negra, não aparecendo na população branca. Causas perinatais também aparecem apenas dentre negros. Em 2016, suicídio tornou-se a terceira causa de óbito que encurta a vida na população parda. Conclusão: As piores condições de saúde na população negra podem refletir as piores condições socioeconômicas dessa população, bem como o racismo institucional e o mito da democracia racial brasileira.

Palavras-chave: Saúde das Minorias Étnicas; Origem Étnica e Saúde; Disparidades nos Níveis de Saúde; Fatores Socioeconômicos; Indicadores de Morbimortalidade
Camila Carvalho de Souza Amorim Matos ${ }^{a}$ Francis Solange Vieira Tourinhob

\footnotetext{
a Secretaria Municipal de Saúde de Florianópolis-SC (SMS-PMF). Florianópolis, SC, Brasil. camilacarvalhoamorim@gmail.com (Autor correspondente)

b Universidade Federal de Santa Catarina (UFSC). Florianópolis, SC, Brasil. francistourinho@gmail.com
}

Como citar: Matos CCSA, Tourinho FSV. Saúde da População Negra: como nascem, vivem e morrem os indivíduos pretos e pardos em Florianópolis (SC). Rev Bras Med Fam Comunidade. 2018;13(40):1-13. http://dx.doi.org/10.5712/rbmfc13(40)1706
Fonte de financiamento: declaram não haver.

Parecer CEP:

2.270.572 (Hospital Governador Celso

Ramos - HGCR), aprovado em 12/09/2017.

Conflito de interesses:

declaram não haver.

Procedência e revisão por pares:

revisado por pares.

Recebido em: 26/02/2018.

Aprovado em: 28/06/2018. 


\begin{abstract}
Objective: To achieve a situational diagnosis of the health conditions of the black population in the city of Florianopolis (SC). Methods: It is a survey-type research, descriptive. Data from the health information systems were consulted in the available databases (municipal electronic records, census data and state and national TABNET DATASUS), analyzing the race/color variable, from 2010 to 2016. Results: The results reveal, among black population, worst schooling and income conditions, higher proportion of adolescent mothers, lower number of prenatal consultations, higher proportions of cases of syphilis in pregnant women and tuberculosis, and lower coverage of private health plans. Black patients attended the Psychosocial Care Centers proportionally more than in the Health Centers. Among the three leading causes of life-shortening deaths, AIDS and homicides appear every year among the black population, not appearing in the white population. Perinatal causes also appear only among black people. In 2016, suicide became the third leading cause of death that shortens life in the brown population. Conclusion: The worst health conditions in the black population may reflect also the worst socioeconomic conditions of this population and the institutional racism and the myth of Brazilian racial democracy.
\end{abstract}

Keywords: Health of Ethnic Minorities; Ethnicity and Health; Health Status Disparities; Socioeconomic Factors; Indicators of Morbidity and Mortality

\title{
Resumen
}

Objetivo: Se pretende realizar un diagnóstico situacional de las condiciones de salud de la población negra en el municipio de Florianópolis (SC). Métodos: Se trata de estudio del tipo compilación, descriptivo. Los datos de los sistemas de información en salud fueron consultados en las bases disponibles (prontuarios electrónicos municipales, datos censales y TABNET DATASUS estadual y nacional), analizando la variable raza/color, en el período de 2010 a 2016. Resultados: Los resultados revelan, en la población negra, peores condiciones de escolaridad y renta, mayor proporción de madres adolescentes, menor número de consultas prenatales, mayores proporciones de casos de sífilis en gestantes y tuberculosis y menor cobertura de plan privado de salud. Los pacientes negros frecuentan proporcionalmente más los Centros de Atención Psicosocial, en comparación a los atendimientos en los Centros de Salud. Entre las tres primeras causas de muertes que acortan la vida, la SIDA y los homicidios aparecen todos los años entre la población negra, no apareciendo en la población blanca. Causas perinatales también aparecen sólo entre negros. En 2016, el suicidio se convirtió en la tercera causa de muerte que acorta la vida en la población parda. Conclusión: Las peores condiciones de salud en la población negra pueden reflejar las peores condiciones socioeconómicas de esa población, bien como el racismo institucional y el mito de la democracia racial brasileña.

Palabras clave: Salud de las Minorías Étnicas; Origen Étnico y Salud; Disparidades en el Estado de Salud; Factores Socioeconómicos; Indicadores de Morbimortalidad

\section{Introdução}

A história do Brasil, construída sobre as bases da desigualdade, reservou a determinados grupos populacionais um lugar de marginalização às políticas públicas, como é o caso da população negra. ${ }^{1,2}$

Lopes $^{3}$ afirma que "indígenas, negros e brancos ocupam lugares desiguais nas redes sociais e trazem consigo experiências também desiguais de nascer, viver, adoecer e morrer". Neste ínterim, a Política Nacional de Saúde Integral da População Negra (PNSIPN) surge como um instrumento de promoção da equidade no âmbito da saúde brasileira. ${ }^{1}$

As diferenças em saúde entre os grupos raciais podem ser encontradas nas mais diversas esferas: menor expectativa de vida na população negra; ${ }^{4,5}$ mais mortes por causas violentas, especialmente entre os jovens, sendo as taxas de homicídio -e feminicídio- crescentes dentre a população negra e decrescentes entre a população branca nos últimos 10 anos; ${ }^{6-8}$ mais mortes por causa materno-infantil, desnutrição infantil e doenças Infectoparasitárias; ${ }^{9}$ e mais mortes por HIVIAIDS. ${ }^{3}$

Sabe-se que, a partir do Censo de 2010, a população negra passou a ser a maioria da população brasileira $(50,7 \%) .{ }^{10}$ Sabe-se, ainda, que $76 \%$ dos atendimentos e $81 \%$ das internações no SUS são de usuários negros e negras. ${ }^{11}$ No estado de Santa Catarina, onde o estudo foi realizado, a população negra correspondia a $7,52 \%$ em 2001 , aumentando para $13,97 \%$ em $2009 .{ }^{10}$ 
O artigo aborda as diferenças nas condições de saúde com base na raça, visando realizar um diagnóstico situacional das condições de saúde da população negra no município de Florianópolis, tendo em vista a baixa produção científica nesta área científica a nível municipal e estadual.

\section{Métodos}

Trata-se de estudo do tipo levantamento descritivo. Os dados secundários foram obtidos a partir de diversas fontes: o Sistema InfoSaúde de prontuários eletrônicos da rede municipal de saúde de Florianópolis; os sistemas de informação disponíveis na plataforma TABNET (Sistema de Informação de Mortalidade -SIM; Sistema de Informação de Nascidos Vivos -SINASC; Sistema de Informação de Agravos de Notificação -SINAN); dados de inquéritos e pesquisas em saúde, também disponíveis no TABNET DATASUS; a plataforma de informação em saúde disponibilizada no sítio do Governo do Estado de Santa Catarina, que fornece dados de indicadores em saúde por município; e dados sociodemográficos disponibilizados pelo Instituto Brasileiro de Geografia e Estatística (IBGE).

As variáveis solicitadas à Gerência de Inteligência e Informação da Prefeitura Municipal de Florianópolis (PMF), dentre os prontuários eletrônicos da rede municipal de saúde, foram todas desagregadas por raça/cor:

1. Número de usuários ativos cadastrados por Centro de Saúde municipal. Foram considerados usuários ativos aqueles que utilizaram o serviço nos últimos três anos.

2. Pessoas com dois ou mais atendimentos no CAPS II - Ponta do Coral, no CAPS Álcool e Drogas Ilha (CAPS AD Ilha) e no CAPS Álcool e Drogas - Continente (CAPS AD Continente), por Centro de Saúde ao qual estão vinculadas, nos últimos três anos.

3. Total de consultas médicas e odontológicas, em um período de 10 anos (2007 a 2016).

4. Exames citopatológicos e de mamografia, por ano, em um período de 10 anos (de 2007 a 2016).

Não foi possível estabelecer a linha cronológica de 10 anos, como previsto, porque houve uma mudança importante na base de dados dos prontuários entre 2009 e 2010. Assim, só foram cedidos às pesquisadoras os dados de 2010 a 2016.

Nos demais sistemas de informação supracitados, procurou-se, em todos os dados disponíveis, a variável raça/cor. Com grande frequência, essa variável não existia ou estava 'ignorada' na quase totalidade dos dados, impossibilitando sua análise.

Dentre os dados dos inquéritos e pesquisas disponíveis no DATASUS, apenas a Pesquisa Nacional de Saúde de 2013 apresentava dados possíveis de serem utilizados.

Os dados são referentes ao município de Florianópolis, capital do estado de Santa Catarina, que apresenta Atenção Primária à Saúde estruturada em Centros de Saúde com Equipes de Saúde da Família, com cobertura populacional de $100 \%$. Conta ainda com quatro policlínicas, três Centros de Atenção Psicossocial (sendo um CAPS II e dois CAPS Álcool e Drogas) e duas Unidades de Pronto Atendimento (UPA). Os hospitais da cidade são de administração estadual. O período de estudo foi 2010 a 2016, quando foi possível estabelecer essa linha cronológica. 
A amostra foi de base populacional com a inclusão de todos os indivíduos negros residentes em Florianópolis. Define-se como negro todo indivíduo com cor de pele preta ou parda.

Os dados secundários foram organizados em um banco de dados em planilhas do programa Microsoft Exce $^{\circledR}$. A análise foi realizada mediante estatística descritiva (frequências absoluta e relativa).

Esta pesquisa foi aprovada pela Comissão de Acompanhamento dos Projetos de Pesquisa em Saúde da Secretaria Municipal de Saúde de Florianópolis e pelo Comitê de Ética em Pesquisa com Seres Humanos do Hospital Governador Celso Ramos pelo parecer nº 2.270.572, conforme Resolução no 466/2012 do Conselho Nacional de Saúde e suas complementares.

\section{Resultados}

Segundo números do IBGE, com base nos dados censitários de 2010, a estimativa populacional para Florianópolis em 2016 era de 477.798 habitantes. Os últimos dados de distribuição populacional por raça/cor são de 2012 , quando a população negra representava $14,67 \%$ da população - 4,95\% preta e 9,72\% parda.

\section{Dados gerados pelo Sistema InfoSaúde}

De acordo com os dados obtidos no sistema de prontuário eletrônico da Prefeitura Municipal de Florianópolis (sistema InfoSaúde), dos usuários ativos nos Centros de Saúde municipais 11,18\% são negros, sendo 4,94\% pretos e 6,24\% pardos. Os cinco Centros de Saúde com maior concentração de usuários ativos negros são: Monte Cristo (35,53\%), Monte Serrat (33,68\%), Vila Aparecida (29,91\%), Novo Continente (24,06\%) e Agronômica (23,49\%).

A porcentagem de usuários negros em consultas médicas e odontológicas realizadas de 2010 a 2016 nos Centros de Saúde foi proporcional à representação dessa população dentre a geral, bem como os números de mamografia e citopatológico do colo do útero solicitados (Tabela 1).

Tabela 1. Número de consultas médicas e odontológicas realizadas nos Centros de Saúde municipais e número de exames de mamografia e citopatológico do colo do útero solicitados nessas unidades, por raça/cor, de 2010 a 2016 , no município de Florianópolis-SC.

\begin{tabular}{|c|c|c|c|c|c|}
\hline Raça/Cor & $\begin{array}{l}\text { Consultas } \\
\text { médicas }\end{array}$ & $\begin{array}{c}\text { Consultas } \\
\text { odontológicas }\end{array}$ & $\begin{array}{l}\text { Mamografia } \\
\text { diagnóstica }\end{array}$ & $\begin{array}{c}\text { Mamografia } \\
\text { rastreamento }\end{array}$ & $\begin{array}{l}\text { Citopatológico } \\
\text { (colo do útero) }\end{array}$ \\
\hline Branca & 4.530 .843 & 271.564 & 6.955 & 63.802 & 110.935 \\
\hline Preta & 294.891 & 22.571 & 488 & 4.271 & 9.245 \\
\hline Parda & 304.106 & 22.835 & 423 & 3.452 & 8.792 \\
\hline Amarela & 32.769 & 1.907 & 60 & 464 & 948 \\
\hline Indígena & 4.866 & 382 & 14 & 78 & 145 \\
\hline Total & 5.168 .019 & 319.295 & 7.940 & 72.069 & 130.066 \\
\hline População negra (\%) & $11,59 \%$ & $14,22 \%$ & $11,47 \%$ & $10,72 \%$ & $13,87 \%$ \\
\hline Variação entre os anos (\%) & 11,31 a 14,46 & 13,57 a 14,81 & 10,06 a 11,83 & 10,14 a 11,40 & 13,42 a 14,42 \\
\hline
\end{tabular}

Fonte: Dados do Sistema InfoSaúde. Tabela construída pela autora. 
Encontrou-se ainda que a porcentagem de pacientes negros nos Centros de Atenção Psicossocial (CAPS) é maior que a proporção geral na rede municipal de saúde. A proporção de negros atendidos no CAPS AD Continente chega a ser o dobro da proporção de negros atendidos nos Centros de Saúde. Também chama a atenção que, apesar de a população preta ser estimada em metade da população parda pelo IBGE, ela representa em todos os CAPS uma porcentagem maior do que a população parda (Tabela 2).

Tabela 2. Usuários ativos negras e negros nos Centros de Saúde e nos CAPS II, CAPS AD Continente e CAPS AD Ilha no município de Florianópolis-SC.

\begin{tabular}{lcccc}
\hline Raça/cor & Centros de Saúde (ESF) & CAPS II & CAPS AD Continente & CAPS AD Ilha \\
\hline Preta (\%) & 4,94 & 8,19 & 11,55 & 7,58 \\
Parda (\%) & 6,24 & 7,88 & 10,45 & 7,58 \\
População negra (\%) & 11,18 & 16,07 & 22 & 15,16 \\
\hline
\end{tabular}

Fonte: Dados do Sistema InfoSaúde. Tabela construída pela autora.

\section{Dados dos sistemas de informação disponíveis no TABNET/DATASUS}

\section{Indicadores e dados básicos}

Apenas os dados demográficos e socioeconômicos contavam com variável raça/cor, sendo os últimos números de 2010.

A taxa de analfabetismo na população geral com idade igual ou superior a 15 anos foi de 1,94\%. Para a população branca, essa taxa era de 1,63\%, enquanto para as populações preta e parda era 3,51 e $3,95 \%$, respectivamente.

Quanto à escolaridade, $60,18 \%$ da população branca possuía fundamental completo ou superior, contra $49,68 \%$ dos pretos e $45,34 \%$ dos pardos. Dentre a população preta, $23,68 \%$ não tinha nenhuma escolaridade ou $1^{\circ}$ fundamental incompleto. Dentre a parda, esse grupo representava $25,37 \%$.

Em 2010, 13,59\% da população preta e 15,32\% da população parda viviam com renda inferior a meio salário mínimo. Esses números eram maiores quando tratava-se de crianças: 29,04\% das crianças pretas e $25,77 \%$ das pardas vivam em situação domiciliar de baixa renda.

A renda média domiciliar per capita era estimada em $\mathrm{R} \$ 1.770,29$ para a população geral, $\mathrm{R} \$ 1.911,99$ para a população branca, $\mathrm{R} \$ 883,41$ para a população preta e $\mathrm{R} \$ 954,11$ para a população parda.

\section{Estatísticas vitais}

Os dados do Sistema de Nascidos Vivos (SINASC) apresentam variável raça/cor, porém, a partir de 2011, todos constam como "ignorados".

Em 2010, a maioria dos nascidos brancos nasceu por parto cesárea $(56,02 \%)$. Já dentre a população negra, a maioria dos pretos $(56,96 \%)$ e pardos $(63,74 \%)$ nasceu de parto vaginal. 
Dentre as mães dos nascidos vivos negros, 70,75\% tinha idade inferior a 29 anos enquanto 41,94\% dos nascidos vivos brancos tinham mãe com mais de 30 anos de idade. A taxa de mães de 10 a 14 anos de idade para a população total foi $0,68 \%$, sendo $0,59 \%$ dentre os nascidos brancos e $3,77 \%$ dentre os nascidos pretos.

Em 2011, a porcentagem de nascidos vivos sem nenhuma consulta pré-natal foi $1,23 \%$ para a população geral; $1,31 \%$ para a população branca; e 3,85\% para a população parda. A maioria dos nascidos brancos teve sete ou mais consultas pré-natal $(71,68 \%)$, contra $48,08 \%$ dos pardos e $61,11 \%$ dos pretos. Quanto à escolaridade materna, em 2010, 45,11\% das mães de nascidos brancos tinha 12 anos ou mais de estudo, contra $25,32 \%$ das mães de nascidos pretos e $21,98 \%$ das de nascidos pardos. A maior porcentagem de mães de nascidos pretos se concentrava entre quatro e sete anos de estudo $(36,71 \%)$.

Entre 2010 e 2016, para o indicador de mortalidade "APVP" (Anos Potenciais de Vida Perdidos), houve diferenças significativas: dentre as três principais causas, predominam dentre a população branca causas como infarto, acidente vascular encefálico (AVE) e câncer. Dentre a população negra, em todos os anos estudados, AIDS ou homicídios eram uma das três principais causas. Chama a atenção que AIDS, homicídios e causas perinatais aparecem apenas dentre as principais causas na população negra. Chama a atenção, ainda, que, em 2016, suicídio torna-se a terceira principal causa de morte APVP dentre a população parda (Quadro 1).

Quadro 1. Principais causas de morte que encurta a vida (APVP), por raça/cor, de 2010 a 2016, em Florianópolis-SC.

\begin{tabular}{|c|c|c|c|c|c|c|c|c|c|}
\hline Ano & \multicolumn{3}{|c|}{$1^{\text {a }}$ causa APVP } & \multicolumn{3}{|c|}{$2^{\mathrm{a}}$ causa APVP } & \multicolumn{3}{|c|}{$3^{\text {a causa APVP }}$} \\
\hline 2010 & DIC & DIC & DIC & DCV & AIDS & Homicídio & $\begin{array}{l}\text { CA de traqueia, } \\
\text { brônquios e } \\
\text { pulmão }\end{array}$ & Homicídio & AIDS \\
\hline 2011 & DIC & DIC & DIC & DCV & AIDS & Homicídio & $\begin{array}{l}\text { CA de traqueia, } \\
\text { brônquios e } \\
\text { pulmão }\end{array}$ & DCV & DCV \\
\hline 2012 & DIC & $\begin{array}{c}\text { DIC } \\
\text { Homicídio }\end{array}$ & Homicídio & DCV & $\begin{array}{l}\text { DM } \\
\text { DCV } \\
\text { PNM }\end{array}$ & DIC & $\begin{array}{l}\text { CA de traqueia, } \\
\text { brônquios e } \\
\text { pulmão }\end{array}$ & Perinatais & $\begin{array}{l}\text { Acidente } \\
\text { de trânsito/ } \\
\text { transporte }\end{array}$ \\
\hline 2013 & DIC & $\begin{array}{c}\text { DIC } \\
\text { AIDS }\end{array}$ & DIC & DCV & DCV & Homicídio & $\begin{array}{l}\text { CA de traqueia, } \\
\text { brônquios e } \\
\text { pulmão }\end{array}$ & Homicídio & $\begin{array}{c}\text { AIDS } \\
\text { Acidente } \\
\text { de trânsito/ } \\
\text { transporte }\end{array}$ \\
\hline 2015 & DIC & DIC & Homicídio & DCV & AIDS & $\begin{array}{l}\text { Acidente } \\
\text { de trânsito/ } \\
\text { transporte }\end{array}$ & PNM & $\begin{array}{c}\text { CA de traqueia } \\
\text { brônquios e } \\
\text { pulmão } \\
\text { Acidente } \\
\text { de trânsito/ } \\
\text { transporte }\end{array}$ & DIC \\
\hline 2016 & DIC & DIC & Homicídio & DCV & PNM & DIC & PNM & Homicídio & $\begin{array}{c}\text { Suicídio } \\
\text { DCV }\end{array}$ \\
\hline
\end{tabular}

DIC: doença isquêmica do coração; DCV: doença cerebrovascular; DM: diabetes mellitus; PNM: pneumonia; CA: câncer. Fonte: Dados do Governo do Estado de Santa Catarina. Quadro construído pela autora. 
Em 2010, para cada óbito APVP na população parda, 22,9 anos de vida foram perdidos. Esse número foi praticamente mantido em 2016: 22,8 anos. Dentre a população branca, perdia-se 11,5 anos potenciais por óbito, em 2010, passando para oito anos em 2016.

\section{Estatísticas epidemiológicas}

Apenas o Sistema de Informação de Agravos de Notificação (SINAN) contava com quesito raça/ cor e dados possíveis de utilização, porém o SINAN-AIDS encontrava-se em manutenção durante todo o processo de coleta de dados.

Os últimos dados de sífilis em gestantes são de 2013 e revelam que as taxas de casos para 100.000 habitantes dentre a população negra são superiores às da população branca. Os dados seguem o mesmo padrão para os casos de tuberculose, cujos últimos dados são de 2015 (Tabela 3).

Tabela 3. Taxa de sífilis em gestantes e tuberculose por 100.000 habitantes $^{\star}$, por ano de diagnóstico ou ano de notificação, por raça/cor em Florianópolis-SC.

\begin{tabular}{lcccc}
\hline & \multicolumn{2}{c}{ Sífilis em gestantes } & \multicolumn{2}{c}{ Tuberculose } \\
\hline & \multicolumn{2}{c}{ Ano de diagnóstico } & \multicolumn{2}{c}{ Ano de notificação } \\
\hline Raça/cor & 2010 & 2013 & 2010 & 2015 \\
Branca & 1,97 & 2,54 & 69,38 & 92,27 \\
Preta & 10,87 & - & 315,47 & 219,14 \\
Parda & - & 4,75 & 100,78 & 95,01 \\
População negra & 3,33 & 3,14 & 166,59 & 136,91 \\
Total & 2,15 & 2,54 & 85,49 & 100,89 \\
\hline
\end{tabular}

Fonte: Dados do TABNET DATASUS. Tabela construída pela autora. * Cálculo com base na população do município por raça/cor nos anos de 2010 e 2012 (último ano disponível).

\section{Dados de inquéritos e pesquisas}

APNS 2013 mostrou que 51,5\% da população branca do município possuía plano de saúde privado, enquanto as taxas para população preta e parda eram, respectivamente, $23,5 \%$ e $30,6 \%$.

Apenas $27,71 \%$ da população branca referiu recorrer à Unidade Básica de Saúde como local de primeiro atendimento quando da necessidade de um serviço de saúde. Para as populações preta e parda, essa porcentagem foi de $59,07 \%$ e $51,10 \%$, respectivamente.

\section{Discussão}

\section{Resumo dos principais achados}

A proporção de pacientes negros atendidos nos CAPS é superior à proporção da população negra geral no município, chegando a representar o dobro dos atendimentos realizados em relação aos Centros de Saúde. A população preta, mesmo equivalendo à metade da parda, corresponde a mais atendimentos nos CAPS do que essa. 
A taxa de analfabetismo é 2,3 vezes maior dentre a população negra em relação à branca e a renda média domiciliar per capita é 2,1 vezes menor na população negra. Mais de $1 / 4$ das crianças negras vivem em situação de pobreza (renda inferior a meio salário mínimo).

Dentre a população branca, predominam os partos cesáreos $(56,02 \%)$ enquanto para a população preta e parda predomina o parto vaginal (56,96\% e $63,74 \%$, respectivamente). Mais de $40 \%$ das mães brancas tinham mais de 30 anos de idade, enquanto $70,75 \%$ das mães negras eram adolescentes e adultas jovens. A taxa de mães de 10 a 14 anos de idade entre pretos é 6,4 vezes maior que entre brancos.

O número de mulheres com nenhuma consulta pré-natal é 2,9 vezes maior dentre os pardos em comparação com os brancos. Mais de $70 \%$ dos brancos apresentaram sete ou mais consultas pré-natal, contra menos da metade dos pardos.

A cada óbito por causa que encurta a vida em uma pessoa parda são perdidos 22,8 anos de vida potenciais; em uma pessoa branca, são perdidos 8 anos. AIDS e homicídios aparecem todos os anos como uma das três primeiras causas de óbitos APVP na população negra. Em 2016, suicídio tornou-se a terceira causa de mortes que encurtam a vida na população parda.

As taxas de sífilis em gestantes são superiores na população negra em relação à branca. A taxa de tuberculose para 100.000 habitantes é 2,37 vezes maior para pretos do que para brancos.

A maioria da população branca tem plano privado de saúde $(51,05 \%)$ e referiu clínica particular como local de primeiro atendimento. A minoria da população negra possui plano privado de saúde $(23,5 \%$ dos pretos e $30,6 \%$ dos pardos). A maioria dos pretos e pardos refere a Unidade Básica de Saúde como local de primeiro atendimento $(59,07 \%$ e $51,10 \%$, respectivamente).

\section{Comparação com a literatura já existente}

As diferenças socioeconômicas e a vulnerabilidade da população negra são conhecidas, ${ }^{4,12}$ como este estudo corroborou. Porém, esses fatores não são suficientes, por si só, para explicar as disparidades existentes, já que fatores como o racismo institucional e o mito da democracia racial refletem no acesso e qualidade dos serviços prestados a essa população..$^{3,13-16}$

Soares, ${ }^{17}$ em discussão sobre renda e raça, mostrou que, se a discriminação de raça e gênero fosse extinta, mulheres negras ganhariam $60 \%$ mais; mulheres brancas $40 \%$ mais; e homens negros 10 a $25 \%$ mais. Esse e outros trabalhos revelam que a heterogeneidade nos níveis de escolaridade não justifica essas diferenças, sugerindo discriminação salarial. ${ }^{17-19}$

A discrepância entre tipo de parto para negras e brancas pode ser explicada por outro dado encontrado neste trabalho: brancos recorrem mais a clínicas privadas, onde são realizadas as maiores taxas de cesariana. ${ }^{20}$

A chance de pré-natal inadequado, ou seja, que não segue as recomendações do Ministério da Saúde para número mínimo de consultas, exames complementares e orientações adequadas à gestante, é conhecidamente maior na população negra, como encontrado neste trabalho, mesmo com a estratificação das características socioeconômicas. Esse fato se reflete em outro dado: causas perinatais aparecem como uma das três principais causas que encurtam a vida da população negra em 2012. A taxa de mortalidade materna é um indicador de falha na assistência obstétrica. Dados das capitais brasileiras mostram taxa de mortalidade materna cerca sete vezes maior dentre mulheres negras em relação às brancas. ${ }^{9,21-23}$ 
O indicador Anos Potenciais de Vida Perdidos/APVP é uma interessante ferramenta para avaliar morte prematura e iniquidades em saúde. ${ }^{24,25} \mathrm{Em}$ São Paulo, um estudo sobre as causas de morte revela que, dentre a população negra, chamam atenção as causas evitáveis, cujo desfecho para óbito o autor considera "estranho, senão repugnante". Por fim, conclui que "a morte negra é uma vida desfeita, e não um fim de vida". ${ }^{26}$

Nos Estados Unidos, estatísticas mostraram que doença cardíaca foi a primeira causa de morte dentre brancos e negros. Chama a atenção que homicídio foi a sétima causa de morte na população negra, não aparecendo dentre as 10 primeiras causas de morte nos demais grupos raciais. ${ }^{27}$

É conhecido que o risco de óbito por homicídio é superior para a população negra, pobre e periférica. ${ }^{6-8,28-30}$ Também já foi relatado que os anos potenciais de vida perdidos nas mortes negras são inúmeras vezes maiores do que nas brancas, como encontrado neste artigo. ${ }^{31}$

São diversas as teorias e hipóteses sobre a violência e seu papel social e político. Jaime AmparoAlves, dissertando sobre que racionalidade justifica (ou até mesmo torna aceitável) a distribuição desigual da morte em certos territórios, traz à tona os conceitos de biopoder (de Michael Foucault) e de necropolítica de (Achilles Mbembe), levantando o racismo como instrumento ideológico de controle populacional.

Traz ainda o conceito de Giorgio Agamben intitulado homo sacer, aquele em situação de permanente exclusão (de humanidade e direitos mínimos de cidadania), que consequentemente também o coloca em uma zona de indistinção entre sacrifício e homicídio, pois sua existência perante as relações de poder está em posição de precariedade. ${ }^{32}$

Alguns estudos associam raça a saúde mental, tendo a população negra maior tendência a esses transtornos, ${ }^{33-35}$ realidade encontrada no fato de suicídio figurar entre as 3 principais causas de morte na população parda em 2016. Nos Estados Unidos, dentre adolescentes negros e negras de 15 a 29 anos, suicídio chega a ser a terceira causa de morte. ${ }^{36} \mathrm{~A}$ desproporção entre a porcentagem de pacientes negros nos Centros de Saúde com relação aos CAPS também pode ser justificada por essa associação (raça e saúde mental).

Considerando que a população branca utiliza mais serviços particulares de saúde, também pode se levantar a hipótese de que utilizem os Centros de Saúde devido à capital estudada ser referência em APS e ter $100 \%$ de cobertura, de qualidade reconhecida; porém não utilizem os CAPS quando da necessidade de atenção à saúde mental, e recorram à rede privada.

Uma possível explicação para a desproporção de pacientes negros no CAPS Álcool e Drogas Continente é o fato de que este Distrito concentra a população negra: três dos cinco Centros de Saúde com mais usuários negros estão no Continente. Porém, diversos trabalhos explicam a propensão da população negra ao envolvimento com drogas, associando à exposição a violência, a discriminação vivida e às maiores condições de vulnerabilidade. ${ }^{34,37,38}$

Outra possibilidade para justificar a desproporção de usuários negros nos CAPS em relação aos Centros de Saúde é a de que essa população esteja tendo menos acesso às unidades básicas, afinal de contas diversos estudos revelam as diferenças no acesso e tratamento recebido nos serviços de saúde, bem como a existência de discriminação nos mesmos. ${ }^{39-41}$ 
Os processos discriminatórios criam limitações aos indivíduos que os vivenciam e podem desencadear problemas de saúde por si só, tendo impactos na autoimagem e no senso de integridade. ${ }^{40,42,43}$ Assim, a exposição ao racismo também é um fator que determina a saúde e o bem-estar - ou a ausência deles, ${ }^{44}$ o que pode explicar por que a população preta (mais fenótipo negro e pele mais escura) frequenta proporcionalmente mais os CAPS do que a população parda.

As taxas de incidência e mortalidade por doenças infecciosas/transmissíveis, como tuberculose e HIVIAIDS, são maiores na população negra, em parte por causa das condições socioeconômicas às quais estão relacionadas. Mulheres e homens negros morrem três vezes mais por tuberculose do que os brancos. ${ }^{9,34}$ Esse fato pode justificar os dados apresentados na Tabela 3 (casos de sífilis em gestantes e tuberculose no município), tratando-se de uma hipótese, tendo em vista que este é um trabalho descritivo. Observa-se que não houve casos de sífilis em gestantes dentre mulheres pardas em 2010 e dentre mulheres pretas em 2013, mas, ainda assim, o dado para mulheres negras supera o mesmo para mulheres brancas. Uma hipótese já antes comentada é a baixa qualidade no preenchimento dos dados nos sistemas de informação.

A mulher negra, que ocupa a mais baixa escala de valorização social, tem fator de risco para exposição ao HIV apenas por ser negra, considerando a maior vulnerabilidade e menor autonomia sobre seu corpo e anticoncepção. ${ }^{45,46}$

Um estudo de 2017 revela o impacto da Estratégia de Saúde da Família sobre a redução da mortalidade por causas evitáveis na população negra após 13 anos de implantação do programa: redução de $27,5 \%$ na mortalidade por doenças infecciosas e de 19,3\% de diabetes na população negra, além de diminuição de $17,9 \%$ nas mortes por deficiências nutricionais e anemia nas crianças menores de cinco anos, ${ }^{47}$ o que sugere o papel essencial da Atenção Primária à Saúde no cuidado integral e na concretização da real equidade em saúde.

\section{Implicações para pesquisa na área/prática profissional}

O trabalho tem implicações diretas para a pesquisa nesta área temática, tendo em vista a necessidade de levantamento de dados. As pesquisas na área ainda são incipientes e os dados ainda são poucos, em quantidade e qualidade. Além disso, por meio da revelação de disparidades em saúde para grupos raciais diferentes, visa-se sensibilizar os profissionais da saúde e gestores para a importância de se debater esse tema.

\section{Fortalezas e limitações}

A principal fortaleza do trabalho é seu vanguardismo, tendo em vista poucos trabalhos na área da saúde foram realizados utilizando variável raça/cor no município de Florianópolis. A pesquisa ampla, incluindo todos os bancos de dados disponíveis, também constitui-se em ponto forte.

A principal limitação foi a ausência da variável raça/cor nos sistemas de informação. Além disso, a forma de classificação racial diferia entre os bancos de dados utilizados: os dados do IBGE contam com autodeclaração; já nos dados do Sistema InfoSaúde e de declarações de nascidos vivos e óbito, por exemplo, geralmente a raça/cor é informada pelo profissional (administrativo ou da saúde) que os preenche. 


\section{Conclusão}

As discrepâncias nas condições de saúde da população negra, quando comparadas com a população geral ou branca, podem ser reflexos não apenas das piores condições socioeconômicas, mas também da maior dificuldade de acesso e permanência nos serviços de saúde, determinada pela discriminação que o racismo institucional e o mito da democracia racial reproduzem. Estudos com metodologia adequada são necessários para avançar na causalidade entre esses fatores.

As dificuldades em produzir dados na área temática de Saúde da População Negra, algumas das quais encontradas nesse trabalho, são muitas: desde a conceituação de "ser negro" e do que é "raça" ou "cor" até a qualificação de preenchimento das fichas que alimentam os sistemas de informação. A própria introdução do quesito raça/cor nos sistemas de informação em saúde brasileiros é muito recente. E, mais grave que isso, muitos deles sequer têm quesito raça/cor até os dias atuais, como é o caso do Sistema de Informação de Atenção Básica (SIAB).

Assim, as iniquidades em saúde aqui apontadas reforçam a necessidade de dados de qualidade e também de maiores pesquisas na área, que embasem a definição de políticas públicas específicas.

Esta pesquisa não contou com nenhum tipo de financiamento externo por órgãos, instituições ou pessoas físicas.

\section{Referências}

1. Brasil. Ministério da Saúde. Secretaria de Gestão Estratégica e Participativa. Política Nacional de Saúde Integral da População Negra: uma política do SUS. $2^{a}$ ed. Brasília: Editora do Ministério da Saúde; 2013 [acesso 2017 Nov 29]. Disponível em: http://bvsms.saude. gov.br/bvs/publicacoes/politica_nacional_saude_integral_populacao.pdf

2. Brasil. Ministério da Saúde. Secretaria Especial de Políticas de Promoção da lgualdade Racial. Caderno de textos básicos do Seminário Nacional de Saúde da População Negra [Internet]. Brasília: Ministério da Saúde; 2004 [acesso 2017 Nov 29]. Disponível em: http://bvsms. saude.gov.br/bvs/publicacoes/seminario_nacional_saude_pop_negra.pdf

3. Lopes F. Experiências desiguais ao nascer, viver, adoecer e morrer: tópicos em saúde da população negra no Brasil. In: Batista LE, Kalckmann S. Seminário Saúde da População Negra Estado de São Paulo 2004. São Paulo, Instituto de Saúde, 2005. p. 53-101.

4. Lopes F. Para além das barreiras dos números: desigualdades raciais e saúde. Cad Saúde Pública. 2005;21(5):1595-601. http://dx.doi. org/10.1590/S0102-311X2005000500034

5. Faustino DM. Equidade racial nas políticas em saúde. In: Batista LE, Werneck J, Lopes F, orgs. Saúde da população negra. Petrópolis: DP et Alii; 2012. p. 98-120.

6. Waiselfisz JJ. Mapa da violência 2015: Adolescentes de 16 e 17 anos do Brasil. Rio de Janeiro: Faculdade Latino-Americana de Ciências Sociais - FLACSO; 2015 .

7. Waiselfisz JJ. Mapa da violência 2015: homicídio de mulheres no Brasil. Rio de Janeiro: Faculdade Latino-Americana de Ciências Sociais - FLACSO; 2015.

8. Waiselfisz JJ. Mapa da violência 2016: homicídios por armas de fogo no Brasil. Rio de Janeiro: Faculdade Latino-Americana de Ciências Sociais - FLACSO; 2015.

9. Brasil. Ministério da Saúde. Secretaria de Vigilância em Saúde. Saúde Brasil 2005: uma análise da situação de saúde no Brasil. Brasília: Ministério da Saúde; 2005.

10. Brasil. Instituto Brasileiro de Geografia e Estatística [homepage na internet]. Indicadores sociais mínimos [acesso 2017 Out 4]. In: Censo 2010. Disponível em: http://www.ibge.gov.br/home/estatistica/populacao/condicaodevida/indicadoresminimos/default_minimos.shtm

11. Pinheiro L, Fontoura N, Prata AC, Soares V. Retrato das desigualdades [publicação na internet]. Brasília: IPEA e UNIFEM; 2006 [acesso 2017 Nov 29]. Disponível em: http://www.ipea.gov.br/retrato/pdf/segundaedicao.pdf 
12. Brasil. Instituto de Pesquisa Econômica Aplicada. Secretaria de Políticas de Promoção da Igualdade Racial. Situação social da população negra por estado [publicação na internet]. Brasília: IPEA; 2014 [acesso 2017 Nov 29]. Disponível em: http://www.seppir.gov.br/centralde-conteudos/publicacoes/pub-pesquisas/situacao-social-da-populacao-negra-por-estado-seppir-e-ipea

13. Munanga K. Superando o racismo na escola. 3a ed. Brasília: Ministério da Educação; 2001.

14. da Cunha EMGP. Recorte étnico-racial: caminhos trilhados e novos desafios. In: Batista LE, Werneck J, Lopes F, orgs. Saúde da população negra. Petrópolis: DP et Alii; 2012. p. 37-48.

15. Munanga K. Cotas para negros. In: Ata do ciclo de debates da Assembléia Legislativa de Minas Gerais. 2012 Maio 13; Belo Horizonte, MG, Brasil.

16. de Araújo EM, Costa MCN, Noronha CV, Hogan VK, Vines Al, de Araújo TM. Desigualdades em saúde e raça/cor da pele: revisão da literatura do Brasil e dos Estados Unidos (1996-2005). Saúde Coletiva [Internet]. 2010 [acesso 2017 Nov 29];7(40):116-21. Disponível em: http://www.redalyc.org/articulo.oa?id=84215105005

17. Soares S. Discriminação de gênero e raça no mercado de trabalho. Bol Mercado Trab Conjunt Anál [Internet]. 2000 [acesso 2017 Nov 29];13:45-51. Disponível em: http://repositorio.ipea.gov.br/bitstream/11058/5729/1/bmt_n.13_discrimina\%C3\%A7\%C3\%A3odege.pdf

18. Martins RB. Desigualdades raciais e políticas de inclusão racial: um sumário da experiência brasileira recente. Santiago: Comisión Económica para América Latina y el Caribe (Serie Políticas Sociales 82) [Internet]; 2004 [acesso 2017 Nov 29]. Disponível em: https:// www.cepal.org/pt-br/publicaciones/6072-desigualdades-raciais-politicas-inclusao-racial-sumario-experiencia-brasileira

19. Lamarca G, Vettore M. A nova composição racial brasileira segundo o Censo 2010. In: Determinantes sociais da saúde: portal e observatório sobre iniquidades em saúde [Internet]. 2012 [acesso 2017 Nov 29]. Disponível em http://dssbr.org/site/2012/01/a-nova-composicaoracial-brasileira-segundo-o-censo-2010/

20.Domingues RMSM, Dias MAB, Nakamura-Pereira M, Torres JA, d'Orsi E, APE, et al. Processo de decisão pelo tipo de parto no Brasil: da preferência inicial das mulheres à via de parto final. Cad Saúde Pública [Internet]. 2014 [acesso 2017 Nov 29];30 Suppl:S101-S116. http://dx.doi.org/10.1590/0102-311X00105113

21. Leal MC, Gama SGN, Pereira APE, Pacheco VE, Carmo CN, Santos RV. A cor da dor: iniquidades raciais na atenção pré-natal e ao parto no Brasil. Cad Saúde Pública [Internet]. 2017 [acesso 2017 Nov 29]; 33(Suppl1):e00078816. http://dx.doi.org/10.1590/0102-311x00078816

22. Howell EA, Zeitlin J, Hebert P, Balbierz A, Egorova N. Paradoxical trends and racial differences in obstetric quality and neonatal and maternal mortality. Obstet Gynecol [Internet]. 2013 [acesso 2017 Nov 29];121(6):1201-8. Disponível em: https://www.ncbi.nlm.nih.gov/ pubmed/23812453

23. Chorl D, Lima CRA. Aspectos epidemiológicos das desigualdades raciais em saúde no Brasil. Cad Saúde Pública [Internet]. 2005 [acesso 2017 Nov 29]; 21(5):1586-94. Disponível em: https://www.scielosp.org/article/csp/2005.v21n5/1586-1594/pt/

24. Johansen JD, Smith E, Juel K, RosdahI N. The Aids epidemic in the city of Copenhagen, Denmark: potential years of life lost and impact on life expectancy. Scand J Public Health [Internet]. 2005 [acesso 2017 Nov 29];33(3):222-7. http://dx.doi.org/10.1080/14034940510005671

25. Lam LT. Different quantitative measures of the impact of premature deaths on the community. Aust N Z J Public Health [Internet]. 2004 [acesso 2017 Nov 29];28(6):555-8. http://dx.doi.org/10.1111/j.1467-842X.2004.tb00046.x

26. Batista LE, Escuder MML, Pereira JCR. A cor da morte: causas de óbito segundo características de raça no Estado de São Paulo, 1999 a 2001. Rev Saúde Pública [Internet]. 2004 [acesso 2017 Nov 29];38(5):630-6. http://dx.doi.org/10.1590/S0034-89102004000500003

27. Heron M. Deaths: leading causes for 2012. National Vital Statistics Reports [Internet]. 2015 [acesso 2017 Nov 29];64(10):1-93. Disponível em: https://www.cdc.gov/nchs/data/nvsr/nvsr64/nvsr64_10.pdf

28. Soares Filho AM, de Souza MFM, Gazal-Carvalho C, Malta DC, Alencar AP, da Silva MMA, et al. Análise da mortalidade por homicídios no Brasil. Epidemiol Serv Saúde [Internet]. 2007 [acesso 2017 Nov 29];16(1):7-18. http://dx.doi.org/10.5123/S1679-49742007000100002

29. Calazans ME, Souza BS, Moitinho KMS, Cardoso CR, Casais Neto R. A espacialização da morte e padrões mórbidos de governança espacial: homicídios de jovens em Salvador 2010-2015 [Internet]. Cad CEAS Rev Crít Humanidades. Salvador: CEAS. 2016 [acesso 2017 Nov 29];238:568-94. Disponível em: https://cadernosdoceas.ucsal.br/index.php/cadernosdoceas/article/view/248

30. Walker GN, McLone SMPH, Mason M, Sheehan K. Rates of firearm homicide by Chicago region, age, sex, and race/ethnicity, 2005-2010. J Trauma Acute Care Surg [Internet]. 2016 [acesso 2017 Nov 29];81(4):S48-S53. Disponível em: https://doi.org/10.1016/j. jemermed.2016.10.036

31. de Araújo EM, Costa MCN, Hogan VK, Mota ELA, de Araújo TM, de Oliveira NF. Diferenciais de raça/cor da pele em anos potenciais de vida perdidos por causas externas. Rev Saúde Pública [Internet]. 2009 [acesso 2017 Nov 29];43(3):405-12. Disponível em: http://www. scielo.br/scielo.php?pid=S0034-89102009000300003\&script=sci_abstract\&tlng=pt 
32. Amparo-Alves J. Necropolítica racial: a produção espacial da morte na cidade de São Paulo. Rev ABPN [Internet]. 2010 [acesso 2017 Nov 29];1(3):89-114. Disponível em: http://abpnrevista.org.br/revista/index.php/revistaabpn1/article/view/276/256

33. Theme Filha MM, Ayers S, da Gama SG, Leal MC. Factors associated with postpartum depressive symptomatology in Brazil: The Birth in Brazil National Research Study, 2011/2012. J Affect Disord [Internet].2016 [acesso 2017 Nov 29];194:159-67. http://dx.doi.org/10.1016/j. jad.2016.01.020

34. Batista LE. Masculinidade, raça/cor e saúde. Ciênc Saúde Coletiva [Internet]. 2005 [acesso 2017 Nov 29];10(1):71-80. http://dx.doi. org/10.1590/S1413-81232005000100013

35. Curtin SC, Warner M, Hedegaard H. Suicide Rates for Females and Males by Race and Ethnicity: United States, 1999 and 2014 [Internet]. Center for Disease Control and Prevention; 2016 [acesso 2017 Nov 29]. Disponível em: https://www.cdc.gov/nchs/data/hestat/suicide/ rates_1999_2014.pdf

36. Crosby A, Molock SD. Suicidal behaviors in the African American Community. J Black Psychol [Internet]. 2006 [acesso 2017 Nov 29];32(3):1-9. http://dx.doi.org/10.1177/0095798406290552

37. de Camargo CL, Santos L, Lacerda RS, TacsiYC. Reflexões sobre a violência vivenciada por crianças e adolescentes negros. In: Batista LE, Werneck J, Lopes F, orgs. Saúde da população negra. Petrópolis: DP et Alii; 2012. p. 305-20.

38. Shield KD, Gmel G, Kehoe-Chan T, Dawson DA, Grant BF, Rehm J. Mortality and potential years of life lost attributable to alcohol consumption by race and sex in the United States in 2005. PLoS One [Internet]. 2013 [acesso 2017 Nov 29];8(1):e51923. http://dx.doi. org/10.1371/journal.pone.0051923

39. Cordeiro RC, Ferreira SL. Discriminação racial e de gênero em discursos de mulheres negras com anemia falciforme. Esc Anna Nery [Internet]. 2009 [acesso 2017 Nov 29];13(2):352-8. http://dx.doi.org/10.1590/S1414-81452009000200016

40. Xavier EC. O olhar das mulheres quilombolas sobre a Atenção Básica e das profissionais das unidades básicas sobre a saúde das comunidades remanescentes de quilombos urbanos de Porto Alegre. In: Batista LE, Werneck J, Lopes F, orgs. Saúde da população negra. Petrópolis: DP et Alii; 2012. p. 193-208.

41. Chehuen Neto JA, Fonseca GM, Brum IV, dos Santos JLCT, Rodrigues TCGF, Paulino KR, et al. Política Nacional de Saúde Integral da População Negra: implementação, conhecimento e aspectos socioeconômicos sob a perspectiva desse segmento populacional. Ciênc Saúde Coletiva [Internet]. 2015 [acesso 2017 Nov 29];20(6):1909-16. http://dx.doi.org/10.1590/1413-81232015206.17212014

42. Clark R, Anderson NB, Clark VR, Williams DR. Racism as a Stressor for African Americans: A Biopsychosocial Model. Am Psychol [Internet]. 1999 [acesso 2017 Nov 29];54(10):805-16. http://dx.doi.org/10.1037/0003-066X.54.10.805

43. Franklin AJ, Boyd-Franklin N. Invisibility Syndrome: A Clinical Model of the Effects of Racism on African American Males. Am J Orthopsychiatr [Internet]. 2000 [acesso 2017 Nov 29];70(1):33-41. http://dx.doi.org/10.1037/h0087691

44. Utsey SO, Chae MH, Brown CF, Kelly D. Effect of ethnic group membership on ethnic identity, race-related stress, and quality of life. Cultur Divers Ethni Minor Psychol [Internet]. 2002 [acesso 2017 Nov 29];8(4):366-77. http://dx.doi.org/10.1037/1099-9809.8.4.367

45. Santos NJS. Mulher e negra: dupla vulnerabilidade às DST/HIV/aids. Saúde Soc. [Internet]. 2016 [acesso 2017 Nov 29];25(3):602-18. http://dx.doi.org/10.1590/s0104-129020162627

46. Souzas R, Marinho OFP, Melo KLO. Acesso à saúde, promoção e prevenção ao HIV/AIDS e o recorte racial/étnico: revisão bibliográfica (1995-2009). In: Batista LE, Werneck J, Lopes F, orgs. Saúde da população negra. Petrópolis: DP et Alii; 2012. p. $266-86$.

47. Hone T, Rasella D, Barreto ML, Majeed A, Millett C. Association between expansion of primary healthcare and racial inequalities in mortality amenable to primary care in Brazil: A national longitudinal analysis. PLoS Med [Internet]. 2017 [acesso 2017 Nov 29];14(5):e1002306. http://dx.doi.org/10.1371/journal.pmed.1002306 